\title{
Mellin Transform of Logarithm and Quotient Function with Reducible Quartic Polynomial in Terms of the Lerch Function
}

\author{
Robert Reynolds*(D) and Allan Stauffer (D) \\ Department of Mathematics and Statistics, York University, Toronto, ON M3J 1P3, Canada; stauffer@yorku.ca \\ * Correspondence: milver@my.yorku.ca
}

\begin{abstract}
A class of definite integrals involving a quotient function with a reducible polynomial, logarithm and nested logarithm functions are derived with a possible connection to contact problems for a wedge. The derivations are expressed in terms of the Lerch function. Special cases are also derived in terms fundamental constants. The majority of the results in this work are new.
\end{abstract}

Keywords: Mellin transform; definite integral; Lerch function; contour integral; Catalan's constant; Glaisher's constant

MSC: Primary 30E20; 33-01; 33-03; 33-04; 33-33B; 33E20; 33E33C

Citation: Reynolds, R.; Stauffer, A. Mellin Transform of Logarithm and Quotient Function with Reducible Quartic Polynomial in Terms of the Lerch Function. Axioms 2021, 10, 236. https://doi.org/10.3390/axioms 10030236

Academic Editor: Hari Mohan Srivastava

Received: 8 August 2021

Accepted: 17 September 2021

Published: 21 September 2021

Publisher's Note: MDPI stays neutral with regard to jurisdictional claims in published maps and institutional affiliations.

Copyright: (C) 2021 by the authors. Licensee MDPI, Basel, Switzerland. This article is an open access article distributed under the terms and conditions of the Creative Commons Attribution (CC BY) license (https:// creativecommons.org/licenses/by/ $4.0 /)$.

\section{Significance Statement}

Mellin transform was first established by Mellin [1]. The theory of this transform is well documented in [2,3]. In this work, we are concerned with extending current literature such as listed in [4] by deriving a new Mellin transform and expressing it in terms of the Lerch function with a possible connection to contact problems for a wedge [5]. We will use this new transform and evaluate it to yield special cases in terms of Catalan's constant $C, \pi$, Euler constant $\gamma$, the zeta function of Riemann $\zeta(s)$, the Hurwitz zeta function $\zeta(s, v)$, and the log-gamma function $\log (\Gamma(x))$. These special cases are new with the aim of providing a new set of integral for use by researchers where applicable.

\section{Introduction}

In this paper, we derive the definite integral given by

$$
\int_{0}^{\infty} \frac{x^{m-1} \log ^{k}(c x)}{\left(a^{2}+x^{2}\right)\left(b^{2}-x^{2}\right)} d x
$$

where the parameters $k, c, a, b, m$ are general complex numbers. The integral will be used to derive special cases in terms of special functions and fundamental constants. The derivations follow the method used by us in [6]. This method involves using a form of the generalized Cauchy's integral formula given by

$$
\frac{y^{k}}{\Gamma(k+1)}=\frac{1}{2 \pi i} \int_{C} \frac{e^{w y}}{w^{k+1}} d w .
$$

where $C$ is in general an open contour in the complex plane where the bilinear concomitant [6] has the same value at the end points of the contour. We then multiply both sides by a function of $x$ and $y$, then take a definite double integral of both sides. This yields a definite integral in terms of a contour integral. Then, we multiply both sides of Equation (2) by another function of $x$ and take the infinite sums of both sides such that the contour integral of both equations are the same. 


\section{Definite Integral of the Contour Integral}

The variable of integration in the contour integral is $\alpha=m+w$. The cut and contour are in the first quadrant of the complex $\alpha$-plane. The cut approaches the origin from the interior of the first quadrant and the contour goes round the origin with zero radius and is on opposite sides of the cut. Using the method in [6] involving Cauchy's integral Equation (2), we replace $y$ by $\log (c x)$ and multiply both sides by $\frac{x^{m-1}}{\left(a^{2}+x^{2}\right)\left(b^{2}-x^{2}\right)}$ to get

$$
\begin{aligned}
& \int_{0}^{\infty} \frac{1}{\Gamma(k+1)} \frac{x^{m-1} \log ^{k}(c x)}{\left(a^{2}+x^{2}\right)\left(b^{2}-x^{2}\right)} d x \\
& =\frac{1}{2 \pi i} \int_{0}^{\infty} \int_{C} \frac{c^{w} w^{-k-1} x^{m+w-1}}{\left(a^{2}+x^{2}\right)\left(b^{2}-x^{2}\right)} d w d x \\
& =\frac{1}{2 \pi i} \int_{C} \int_{0}^{\infty} \frac{c^{w} w^{-k-1} x^{m+w-1}}{\left(a^{2}+x^{2}\right)\left(b^{2}-x^{2}\right)} d x d w \\
& =\frac{1}{2 \pi i} \int_{C} \frac{\pi c^{w} w^{-k-1} \csc \left(\frac{1}{2} \pi(m+w)\right)\left(b^{2} a^{m+w}+a^{2} b^{m+w} \cos \left(\frac{1}{2} \pi(m+w)\right)\right)}{2 a^{2} b^{2}\left(a^{2}+b^{2}\right)} d w
\end{aligned}
$$

from Equation (3.264.1) in [7] where $0<\operatorname{Re}(w+m), \operatorname{Re}(a)>0, \operatorname{Re}(b)>0$. We are able to switch the order of integration over $w+m$ and $x$ using Fubini's theorem since the integrand is of bounded measure over the space $\mathbb{C} \times[0, \infty)$.

\section{The Lerch Function}

The Lerch function see Section (1.11) in [8], also popularly called the Hurwitz-Lerch function $[9,10]$, has a series representation given by

$$
\Phi(z, s, v)=\sum_{n=0}^{\infty}(v+n)^{-s} z^{n}
$$

where $|z|<1, v \neq 0,-1, \ldots$ and is continued analytically by its integral representation given by

$$
\Phi(z, s, v)=\frac{1}{\Gamma(s)} \int_{0}^{\infty} \frac{t^{s-1} e^{-v t}}{1-z e^{-t}} d t=\frac{1}{\Gamma(s)} \int_{0}^{\infty} \frac{t^{s-1} e^{-(v-1) t}}{e^{t}-z} d t
$$

where $\operatorname{Re}(v)>0$, and either $|z| \leq 1, z \neq 1, \operatorname{Re}(s)>0$, or $z=1, \operatorname{Re}(s)>1$.

\section{Infinite Sum of the Contour Integral}

\subsection{Derivation of the First Contour Integral}

In this section, we will derive the contour integral given by

$$
\frac{1}{2 \pi i} \int_{C} \frac{\pi c^{w} w^{-k-1} b^{m+w-2} \cot \left(\frac{1}{2} \pi(m+w)\right)}{2\left(a^{2}+b^{2}\right)} d w
$$


Again, using the method in [6] and Equation (2), we replace $y$ by $\log (b)+\log (c)+$ $i \pi(y+1)$ multiply both sides by $-\frac{i \pi b^{m-2} e^{i \pi m(y+1)}}{a^{2}+b^{2}}$ and take the infinite sum of both sides over $y \in[0, \infty)$ simplifying in terms the Lerch function to get

$$
\begin{aligned}
& -\frac{(i \pi)^{k+1} e^{i \pi m} b^{m-2} \Phi\left(e^{i m \pi},-k, 1-\frac{i(\log (b)+\log (c))}{\pi}\right)}{\Gamma(k+1)\left(a^{2}+b^{2}\right)} \\
& =-\frac{1}{2 \pi i} \sum_{y=0}^{\infty} \int_{C} \frac{i \pi b^{m-2} w^{-k-1} \exp (w(\log (b)+\log (c)+i \pi(y+1))+i \pi m(y+1))}{a^{2}+b^{2}} d w \\
& =-\frac{1}{2 \pi i} \int_{C} \sum_{y=0}^{\infty} \frac{i \pi b^{m-2} w^{-k-1} \exp (w(\log (b)+\log (c)+i \pi(y+1))+i \pi m(y+1))}{a^{2}+b^{2}} d w \\
& =\frac{1}{2 \pi i} \int_{C} \frac{\pi c^{w} w^{-k-1} b^{m+w-2}\left(\cot \left(\frac{1}{2} \pi(m+w)\right)+i\right)}{2\left(a^{2}+b^{2}\right)} d w
\end{aligned}
$$

similar to Equation (1.232.1) in [7] where

$$
\cot (x)=-2 i \sum_{y=0}^{\infty} e^{2 x i(y+1)}-i
$$

\subsection{Derivation of the Second Contour Integral}

In this section, we will derive the contour integral given by

$$
-\frac{1}{2 \pi i} \int_{C} \frac{\pi c^{w} w^{-k-1} a^{m+w-2} \csc \left(\frac{1}{2} \pi(m+w)\right)}{2\left(a^{2}+b^{2}\right)} d w
$$

Again, using the method in [6] and Equation (2), we replace $y$ by $\log (a)+\log (c)+$ $\frac{1}{2} i \pi(2 y+1)$ multiply both sides by $-\frac{i \pi a^{m-2} e^{\frac{1}{2} i \pi m(2 y+1)}}{a^{2}+b^{2}}$ and take the infinite sum of both sides over $y \in[0, \infty)$ simplifying in terms the Lerch function to get

$$
\begin{aligned}
- & \frac{(i \pi)^{k+1} e^{\frac{i \pi m}{2}} a^{m-2} \Phi\left(e^{i m \pi},-k, \frac{-2 i \log (a)-2 i \log (c)+\pi}{2 \pi}\right)}{\Gamma(k+1)\left(a^{2}+b^{2}\right)} \\
& =-\frac{1}{2 \pi i} \sum_{y=0}^{\infty} \int_{C} \frac{i \pi c^{w} w^{-k-1} a^{m+w-2} e^{\frac{1}{2} i \pi(2 y+1)(m+w)}}{a^{2}+b^{2}} d w \\
& =-\frac{1}{2 \pi i} \int_{C} \sum_{y=0}^{\infty} \frac{i \pi c^{w} w^{-k-1} a^{m+w-2} e^{\frac{1}{2} i \pi(2 y+1)(m+w)}}{a^{2}+b^{2}} d w \\
& =\frac{1}{2 \pi i} \int_{C} \frac{\pi c^{w} w^{-k-1} a^{m+w-2} \csc \left(\frac{1}{2} \pi(m+w)\right)}{2\left(a^{2}+b^{2}\right)} d w
\end{aligned}
$$

from Equation (1.232.2) in [7] where $\operatorname{Im}(w+m)>0$ in order for the sum to converge.

Derivation of the Additional Contour Integral

Using the method in [6] and Equation (2), we replace $y \rightarrow \log (b)+\log (c)$ multiply both sides by $\frac{i \pi b^{m-2}}{2\left(a^{2}+b^{2}\right)}$ and simplify to get

$$
\frac{i \pi b^{m-2}(\log (b)+\log (c))^{k}}{2 \Gamma(k+1)\left(a^{2}+b^{2}\right)}=\frac{1}{2 \pi i} \int_{C} \frac{i \pi c^{w} w^{-k-1} b^{m+w-2}}{2\left(a^{2}+b^{2}\right)} d w
$$

\section{Definite Integral in Terms of the Lerch Function}

The proceeding theorem is an instant consequence of the previous sections. 
Theorem 1. For all $k, c, a, b \in \mathbb{C}$,

$$
\begin{aligned}
\int_{0}^{\infty} \frac{x^{m-1} \log ^{k}(c x)}{\left(a^{2}+x^{2}\right)\left(b^{2}-x^{2}\right)} d x= & -\frac{(i \pi)^{k+1} e^{\frac{i \pi m}{2} a^{m-2} \Phi\left(e^{i m \pi},-k, \frac{-2 i \log (a)-2 i \log (c)+\pi}{2 \pi}\right)}}{a^{2}+b^{2}} \\
& -\frac{(i \pi)^{k+1} e^{i \pi m} b^{m-2} \Phi\left(e^{i m \pi},-k, 1-\frac{i(\log (b)+\log (c))}{\pi}\right)}{a^{2}+b^{2}} \\
& -\frac{i \pi b^{m-2}(\log (b)+\log (c))^{k}}{2\left(a^{2}+b^{2}\right)}
\end{aligned}
$$

Main Results

In the proceeding section, we will evaluate Equation (12) and simplify the Lerch function in terms of the special functions and fundamental constants stated.

Derivation of Entry (3.264.1) in [7]

Proposition 1. For all $0<\operatorname{Re}(m)<4, a>0, b>0$,

$$
\int_{0}^{\infty} \frac{x^{m-1}}{\left(a^{2}+x^{2}\right)\left(b^{2}-x^{2}\right)} d x=\frac{\pi a^{2} b^{m} \cot \left(\frac{\pi m}{2}\right)+\pi b^{2} a^{m} \csc \left(\frac{\pi m}{2}\right)}{2 a^{4} b^{2}+2 a^{2} b^{4}}
$$

Proof. Use Equation (12) and set $k=0$ and simplify using entry (2) below Table (64:12:7) in [11].

\section{Proposition 2.}

$$
\begin{aligned}
& \int_{0}^{\infty} \frac{\log ^{k}(c x)}{\left(a^{2}+x^{2}\right)\left(b^{2}-x^{2}\right)} d x \\
& =\frac{1}{2 a b\left(a^{2}+b^{2}\right)} e^{\frac{i \pi k}{2}}(2 \pi)^{k+1}\left(i a \left(\zeta\left(-k, \frac{-i \log (b)-i \log (c)+\pi}{2 \pi}\right)\right.\right. \\
& \left.-\zeta\left(-k, \frac{-i \log (b)-i \log (c)+2 \pi}{2 \pi}\right)\right)+b\left(\zeta\left(-k, \frac{-2 i \log (a)-2 i \log (c)+\pi}{4 \pi}\right)\right. \\
& \left.\left.-\zeta\left(-k, \frac{-2 i \log (a)-2 i \log (c)+3 \pi}{4 \pi}\right)\right)\right)-i \pi a(\log (b)+\log (c))^{k}
\end{aligned}
$$

Proof. Use Equation (12) and set $m=1$ and simplify in terms of the Hurwitz zeta function $\zeta(s, a)$ using entry (4) below Table (64:12:7) in [11].

\section{Proposition 3.}

$$
\begin{aligned}
& \int_{0}^{\infty} \frac{\log ^{k}(c x)}{\left(a^{2}+x^{2}\right)\left(c^{2} x^{2}-1\right)} d x \\
& =\frac{1}{a^{3} c^{2}+a} e^{\frac{i \pi k}{2}} \pi^{k+1}\left(-2^{k} \zeta\left(-k, \frac{-2 i \log (a)-2 i \log (c)+\pi}{4 \pi}\right)\right. \\
& \left.+2^{k} \zeta\left(-k, \frac{-2 i \log (a)-2 i \log (c)+3 \pi}{4 \pi}\right)+i a c\left(2^{k+1}-1\right) \zeta(-k)\right)
\end{aligned}
$$

Proof. Use Equation (14) and set $b=1 / c$ and simplify.

\section{Proposition 4.}

$$
\int_{0}^{\infty} \frac{\log ^{k}(x)}{x^{4}-1} d x=-\frac{1}{2} e^{\frac{i \pi k}{2}} \pi^{k+1}\left(2^{k}\left(\zeta\left(-k, \frac{1}{4}\right)-\zeta\left(-k, \frac{3}{4}\right)-2 i \zeta(-k)\right)+i \zeta(-k)\right)
$$

Proof. Use Equation (15) and set $a=c=1$ and simplify. 


\section{Example 1.}

$$
\int_{0}^{\infty} \frac{\log (x) \log (\log (x))}{x^{4}-1} d x=\frac{1}{48} \pi\left(\pi\left(-72 \log (A)+6+3 i \pi+\log \left(256 \pi^{6}\right)\right)+24 i C\right)
$$

Proof. Use Equation (16) take the first partial derivative with respect to $k$ and set $k=1$ and simplify in terms of Catalan's constant $C$, Glaisher's constant $A$ and $\pi$ using Equation (3:12:4) in [11] and Equation (3.13) in [12].

\section{Example 2.}

$$
\begin{aligned}
\int_{0}^{\infty} \frac{\log ^{2}(x) \log (\log (x))}{x^{4}-1} d x & =\frac{1}{32}\left(-2 \pi^{3}\left(32 \zeta^{\prime}\left(-2, \frac{1}{4}\right)-32 \zeta^{\prime}\left(-2, \frac{3}{4}\right)\right.\right. \\
& \left.+\log (2)+\log (\pi))-28 i \pi \zeta(3)-i \pi^{4}\right)
\end{aligned}
$$

Proof. Use Equation (16) take the first partial derivative with respect to $k$ and set $k=2$ and simplify.

\section{Example 3.}

$$
\begin{array}{r}
\int_{0}^{\infty} \frac{\sqrt{\log (x)} \log (\log (x))}{x^{4}-1} d x=\frac{1}{4} \sqrt[4]{-1} \pi^{3 / 2}\left(2 \sqrt{2}\left(\zeta^{\prime}\left(-\frac{1}{2}, \frac{1}{4}\right)-\zeta^{\prime}\left(-\frac{1}{2}, \frac{3}{4}\right)\right)\right. \\
-2 i(2 \sqrt{2}-1) \zeta^{\prime}\left(-\frac{1}{2}\right) \\
-i \sqrt{2}\left(\zeta\left(-\frac{1}{2}, \frac{1}{4}\right)-\zeta\left(-\frac{1}{2}, \frac{3}{4}\right)\right)\left(\pi-i \log \left(4 \pi^{2}\right)\right) \\
+\left(\frac{1}{\sqrt{2}}-\frac{1}{4}\right) \zeta\left(\frac{3}{2}\right) \\
\left.+\frac{i \zeta\left(\frac{3}{2}\right)(\log (\pi)-2 \sqrt{2} \log (2 \pi))}{2 \pi}\right)
\end{array}
$$

Proof. Use Equation (16) take the first partial derivative with respect to $k$ and set $k=1 / 2$ and simplify.

\section{Proposition 5.}

$$
\begin{aligned}
& \int_{0}^{\infty} \frac{x^{m-1} \sqrt{\log (x)}}{\left(x^{2}-1\right)\left(a^{2}+x^{2}\right)} d x \\
& =\frac{1}{a^{4}+a^{2}}(-1)^{3 / 4} \pi^{3 / 2}\left(e^{\frac{i \pi m}{2}} a^{m} \Phi\left(e^{i m \pi},-\frac{1}{2}, \frac{1}{2}-\frac{i \log (a)}{\pi}\right)+a^{2} L i_{-\frac{1}{2}}\left(e^{i m \pi}\right)\right)
\end{aligned}
$$

Proof. Use Equation (12) and set $k=1 / 2, c=b=1$ and simplify using Equation (64:12:2) in [11]. 


\section{Proposition 6.}

$$
\begin{aligned}
& \int_{0}^{\infty} \frac{\left(x^{p}-x^{m}\right) \log ^{k}(c x)}{x\left(a^{2}+x^{2}\right)^{2}\left(c^{2} x^{2}-1\right)} d x \\
& =\frac{\pi^{k}}{2 a^{4}\left(a^{2} c^{2}+1\right)^{2}}\left(i e ^ { \frac { i \pi k } { 2 } } \left(\pi e ^ { \frac { i \pi m } { 2 } } a ^ { m } \left(a^{2} c^{2}(m-4)\right.\right.\right. \\
& +m-2) \Phi\left(e^{i m \pi},-k, \frac{-2 i \log (a)-2 i \log (c)+\pi}{2 \pi}\right) \\
& +i k e^{\frac{i \pi p}{2}}\left(a^{2} c^{2}+1\right) a^{p} \Phi\left(e^{i p \pi}, 1-k, \frac{-2 i \log (a)-2 i \log (c)+\pi}{2 \pi}\right) \\
& -\pi e^{\frac{i \pi p}{2}} a^{p}\left(a^{2} c^{2}(p-4)+p-2\right) \Phi\left(e^{i p \pi},-k, \frac{-2 i \log (a)-2 i \log (c)+\pi}{2 \pi}\right) \\
& \left.-2 \pi a^{4}\left(\frac{1}{c}\right)^{m-4} L i_{-k}\left(e^{i m \pi}\right)+2 \pi a^{4}\left(\frac{1}{c}\right)^{p-4} \operatorname{Li}-k\left(e^{i p \pi}\right)\right) \\
& \left.+k\left(a^{2} c^{2}+1\right) a^{m} e^{\frac{1}{2} i \pi(k+m)} \Phi\left(e^{i m \pi}, 1-k, \frac{-2 i \log (a)-2 i \log (c)+\pi}{2 \pi}\right)\right)
\end{aligned}
$$

Proof. Use Equation (12) take the first partial derivative with respect to $a$ and set $b=1 / c$. Next form a second equation by replacing $m \rightarrow p$ and take their difference and simplify.

\section{Example 4.}

$$
\begin{aligned}
& \int_{0}^{\infty} \frac{1-\sqrt{x}}{\left(x^{2}+1\right)\left(x^{2}+(-1)^{2 / 3}\right)^{2} \log (i x)} d x \\
& =\frac{-24}{144 \pi}\left((-1)^{5 / 12}+(-1)^{3 / 4}\right) \Phi\left(-i, 2, \frac{4}{3}\right)+4 \pi\left(59 i-45(-1)^{5 / 12}-9(-1)^{3 / 4}\right. \\
& \left.+18(-1)^{5 / 6}+\left(3+3(-1)^{3 / 4}-7 i \sqrt{3}+(2+2 i) \sqrt{6}\right) \pi\right) \\
& +\log \left(2^{6\left(-18 i-\sqrt[4]{-1}+4(-1)^{7 / 12}+5(-1)^{11 / 12}+6 \sqrt{3}\right) \pi}\right. \\
& \left.(2-\sqrt{3})^{-6(-1)^{7 / 12}(5+\sqrt[3]{-1}) \pi}(2+\sqrt{3})^{6 \sqrt[4]{-1}(5+\sqrt[3]{-1}) \pi}\right) \\
& +(9+3 i \sqrt{3})\left(\psi^{(1)}\left(\frac{2}{3}\right)-\psi^{(1)}\left(\frac{7}{6}\right)\right)
\end{aligned}
$$

Proof. Use Equation (21) and set $k=-1, c=i, a=e^{\pi i / 3}, m=1, p=3 / 2$ and simplify using entry (2) in Table below (64:12:2) in [11].

\section{Example 5.}

$$
\begin{aligned}
& \int_{0}^{\infty} \frac{1-\sqrt{x}}{\left(x^{2}+i\right)^{2}\left(x^{2}+1\right) \log (i x)} d x \\
& =\frac{1}{16 \pi}\left((4-4 i) \sqrt[8]{-1} \Phi\left(-i, 2, \frac{5}{4}\right)-2(\sqrt[4]{-1}+(-4+2 i)) \pi^{2}\right. \\
& +i \sqrt{2}\left(\psi^{(1)}\left(\frac{5}{8}\right)-\psi^{(1)}\left(\frac{9}{8}\right)\right)+4 \pi((2-10 i) \sqrt[8]{-1}-(8-4 i) \sqrt{2} \\
& +(-1)^{3 / 4} \log (2)+\log (4)+(2+3 i) \sqrt{2} \tanh ^{-1}\left((-1)^{3 / 8}\right) \\
& \left.\left.+(3-2 i) \sqrt{2} \tanh ^{-1}\left((-1)^{7 / 8}\right)+(4-2 i) \operatorname{coth}^{-1}(\sqrt{2})\right)\right)
\end{aligned}
$$

Proof. Use Equation (21) and set $k=-1, c=i, a=e^{\pi i / 4}, m=1, p=3 / 2$ and simplify using entry (3) in Table below (64:12:2) in [11]. 


\section{Example 6.}

$$
\begin{aligned}
& \int_{0}^{\infty} \frac{\sqrt{x}-1}{\left(x^{2}+1\right)\left(x^{2}+4\right)^{2} \log (i x)} d x \\
& =\frac{\pi}{576 \pi}\left(-44 i \Phi\left(-1,1,1-\frac{i \log (2)}{\pi}\right)\right. \\
& -(38-38 i) \Phi\left(-i, 1,1-\frac{i \log (2)}{\pi}\right) \\
& -64 \sqrt[4]{-1} \log (1+i)+64 i \log (2)) \\
& +3\left((4+4 i) \Phi\left(-i, 2,1-\frac{i \log (2)}{\pi}\right)\right. \\
& \left.-\psi^{(1)}\left(\frac{\pi-i \log (2)}{2 \pi}\right)+\psi^{(1)}\left(1-\frac{i \log (2)}{2 \pi}\right)\right)
\end{aligned}
$$

Proof. Use Equation (21) and set $k=-1, c=i, a=2, m=1, p=3 / 2$ and simplify.

\section{Example 7.}

$$
\int_{0}^{\infty} \frac{\log (\log (2 i x))}{\left(x^{2}+1\right)\left(4 x^{2}+1\right)} d x=\frac{1}{12} \pi\left(i \pi+\log \left(\frac{4(\pi+i \log (2))^{4} \Gamma\left(-\frac{\pi+i \log (2)}{2 \pi}\right)^{4}}{\pi^{2} \log ^{4}(2) \Gamma\left(-\frac{i \log (2)}{2 \pi}\right)^{4}}\right)\right)
$$

Proof. Use (15) take the first partial derivative with respect to $k$ then set $k=0, c=2 i, a=1$ and simplify using Equation (64:10:2) in [11].

\section{Proposition 7.}

$$
\begin{array}{r}
\int_{0}^{\infty} \frac{\log (\log (b x))}{\left(a^{2}+x^{2}\right)\left(1-b^{2} x^{2}\right)} d x=\frac{\pi}{4\left(a^{3} b^{2}+a\right)}\left(4 \log \Gamma\left(-\frac{2 i \log (a)+2 i \log (b)+\pi}{4 \pi}\right)\right. \\
-4 \log \Gamma\left(-\frac{2 i \log (a)+2 i \log (b)+3 \pi}{4 \pi}\right)-\pi a b \\
+2 i a b \log (2)-4 \log (-2 i \log (a)-2 i \log (b)-3 \pi) \\
+4 \log (-2 i \log (a)-2 i \log (b)-\pi)+i \pi+2 \log (\pi)+\log (4))
\end{array}
$$

Proof. Use (15) take the first partial derivative with respect to $k$ then set $k=0, c=b$ and simplify using Equation (64:10:2) in [11].

\section{Example 8.}

$$
\begin{aligned}
\int_{0}^{\infty} & \frac{\log \left(\log (x)+\frac{i \pi}{2}\right)}{\left(x^{2}+1\right)\left(x^{2}+4\right)} d x=-\frac{1}{24} \pi\left(-i \pi+\log \left(\frac{\pi^{2}}{4}\right)\right. \\
& \left.+4 \log \left(-\frac{2 i \log (2) \Gamma\left(-\frac{i \log (2)}{2 \pi}\right)}{(-2 \pi-2 i \log (2)) \Gamma\left(-\frac{2 \pi+2 i \log (2)}{4 \pi}\right)}\right)\right)
\end{aligned}
$$

Proof. Use (26) set $a=2, b=i$ and simplify. 


\section{Proposition 8.}

$$
\begin{array}{r}
\int_{0}^{\infty} \frac{\log (b x) \log (\log (b x))}{\left(a^{2}+x^{2}\right)\left(1-b^{2} x^{2}\right)} d x \\
=\frac{\pi}{24\left(a^{3} b^{2}+a\right)}\left(-48 i \pi\left(\zeta^{\prime}\left(-1, \frac{-2 i \log (a)-2 i \log (b)+\pi}{4 \pi}\right)\right.\right. \\
\left.-\zeta^{\prime}\left(-1, \frac{-2 i \log (a)-2 i \log (b)+3 \pi}{4 \pi}\right)\right) \\
+\pi(a b(72 \log (A)-6-3 i \pi-8 \log (2)-6 \log (\pi))+6 i \log (a)+6 i \log (b)) \\
+12 \log (2 \pi)(\log (a)+\log (b)))
\end{array}
$$

Proof. Use Equation (15) take the first partial derivative with respect to $k$ then set $k=1$, $c=b$ and simplify using Equation (3.13) in [12].

\section{Example 9.}

$$
\int_{0}^{\infty} \frac{\log (x) \log (\log (x))}{1-x^{4}} d x=\frac{1}{48} \pi\left(\pi\left(\log \left(\frac{A^{72}}{256 e^{6} \pi^{6}}\right)-3 i \pi\right)-24 i C\right)
$$

Proof. Use Equation (26) and set $a=b=1$ and simplify in terms of Glaisher's $A$ and Catalan $C$ constants using Equation (3.13) in [12].

\section{Example 10.}

$$
\begin{aligned}
& \int_{0}^{\infty} \frac{\log (x) \log (\log (x))}{\left(1-x^{2}\right)\left(x^{2}+4\right)} d x \\
& =\frac{1}{240} \pi\left(-48 i \pi\left(\zeta^{\prime}\left(-1, \frac{\pi-2 i \log (2)}{4 \pi}\right)\right.\right. \\
& \left.-\zeta^{\prime}\left(-1, \frac{3 \pi-2 i \log (2)}{4 \pi}\right)\right) \\
& +\pi(2(72 \log (A)-6-3 i \pi-8 \log (2)-6 \log (\pi)) \\
& +6 i \log (2))+12 \log (2) \log (2 \pi))
\end{aligned}
$$

Proof. Use Equation (26) and set $a=2, b=1$ and simplify in terms of Glaisher's $A$ constant using Equation (3.13) in [12].

\section{Example 11.}

$$
\begin{aligned}
& \int_{0}^{\infty} \frac{\log (2 x) \log (\log (2 x))}{16 x^{4}-1} d x \\
& =\frac{1}{96} \pi\left(\pi\left(-72 \log (A)+6+3 i \pi+\log \left(256 \pi^{6}\right)\right)+24 i C\right)
\end{aligned}
$$

Proof. Use Equation (26) and set $a=2, b=1 / 2$ and simplify in terms of Glaisher's $A$ and Catalan's C constants using Equation (3.13) in [12].

\section{Example 12.}

$$
\begin{array}{r}
\int_{0}^{\infty} \frac{\log \left(\log (x)+\frac{i \pi}{2}\right)}{\left(x^{2}+1\right)\left(x^{2}+3\right)} d x=\frac{\pi}{8 \sqrt{3}}(-i \pi+i \sqrt{3} \pi+\sqrt{3} \log (4) \\
\left.+4 \log \left(\frac{(\log (3)-2 i \pi) \Gamma\left(-\frac{1}{2}-\frac{i \log (3)}{4 \pi}\right)}{\sqrt{2 \pi} \log (3) \Gamma\left(-\frac{i \log (3)}{4 \pi}\right)}\right)\right)
\end{array}
$$

Proof. Use Equation (26) and set $a=\sqrt{3}, c=e^{\pi i / 2}$ and simplify. 


\section{Example 13.}

$$
\begin{array}{r}
\int_{0}^{\infty} \frac{\sqrt{\log (2 x)}}{4 x^{4}+3 x^{2}-1} d x=\left(-\frac{1}{5}-\frac{i}{5}\right) \pi^{3 / 2}\left(\zeta\left(-\frac{1}{2}, \frac{\pi-2 i \log (2)}{4 \pi}\right)\right. \\
\left.-\zeta\left(-\frac{1}{2}, \frac{3}{4}-\frac{i \log (2)}{2 \pi}\right)+i(\sqrt{2}-4) \zeta\left(-\frac{1}{2}\right)\right)
\end{array}
$$

Proof. Use Equation (12) and set $k=1 / 2, c=2, m=a=1, b=1 / 2$ and simplify using entry (4) in Table below (64:12:7) in [11].

\section{Discussion}

In this article, using our contour integral method [6] we derived definite integrals using the Lerch function. We were able to provide formulae and extend the range of computation through analytic continuation of the Lerch function. We will be applying our method to other integrals to derive other known and new integral forms in terms of other special functions. The results in this work were numerically verified using Wolfram Mathematica for complex ranges of the parameters.

Author Contributions: Conceptualization, R.R.; Funding acquisition, A.S.; Supervision, A.S. All authors have read and agreed to the published version of the manuscript.

Funding: This research is supported by NSERC Canada under Grant 504070.

Institutional Review Board Statement: Not applicable.

Informed Consent Statement: Not applicable.

Data Availability Statement: Not applicable.

Conflicts of Interest: The authors declare no conflict of interest.

\section{References}

1. Melin, H. Uber den zusamenhang zwischen den linearen differential-und diferenzen-gleichungen. Acta Math. 1902, 25, 139-164. [CrossRef]

2. Doetsch, G. Handbuch der Laplace Transformation; Birkhauser: Basel, Switzerland, 1950-1956; Volumes 1-3.

3. Titchmarsh, E.G. Introduction to the Theory of Fourier Integrals; Clarendon: Oxford, UK, 1937; 390p.

4. Oberhettinger, F. Tables of Mellin Transforms; Springer: Berlin/Heidelberg, Germany, 1974; 278p.

5. Alexandrov, V.M.; Pozharskii, D.A. Contact Problems for a Wedge. In Three-Dimensional Contact Problems; Solid Mechanics and Its Applications; Springer: Dordrecht, The Netherlands, 2001; Volume 93. [CrossRef]

6. Reynolds, R.; Stauffer, A. A Method for Evaluating Definite Integrals in Terms of Special Functions with Examples. Int. Math. Forum 2020, 15, 235-244. [CrossRef]

7. Gradshteyn, I.S.; Ryzhik, I.M. Tables of Integrals, Series and Products, 6th ed.; Academic Press: Cambridge, MA, USA, 2000.

8. Erdéyli, A.; Magnus, W.; Oberhettinger, F.; Tricomi, F.G. Higher Transcendental Functions; McGraw-Hill Book Company, Inc.: New York, NY, USA; Toronto, ON, Canada; London, UK, 1953; Volume I.

9. Srivastava, H.M. Some general families of the Hurwitz-Lerch Zeta functions and their applications: Recent developments and directions for further researches. Proc. Inst. Math. Mech. Natl. Acad. Sci. Azerbaijan 2019, 45, 234-269. [CrossRef]

10. Srivastava, H.M. The Zeta and Related Functions: Recent Developments. J. Adv. Eng. Comput. 2019, 3, 329-354. [CrossRef]

11. Oldham, K.B.; Myland, J.C.; Spanier, J. An Atlas of Functions: With Equator, the Atlas Function Calculator, 2nd ed.; Springer: New York, NY, USA, 2009.

12. Choi, J.; Srivastava, H.M. A certain family of series associated with the Zeta and related functions. Hiroshima Math. J. 2002, 32, 417-429. [CrossRef] 